\title{
Shear Stress Sensing with Elastic Microfence Structures
}

\author{
Alexxandra Cisotto ${ }^{1}$, Frank L. Palmieri' ${ }^{2}$, Aditya Saini ${ }^{3}$, Yi Lin $^{4}$, Christopher S. Thurman ${ }^{5}$, Jinwook Kim ${ }^{3}$, \\ Taeyang Kim ${ }^{3}$, John W. Connell ${ }^{2}$, Yong $\mathrm{Zhu}^{3}$, Ashok Gopalarathnam ${ }^{3}$, Xiaoning Jiang ${ }^{3}$, Christopher J. \\ Wohl $^{2}$ \\ ${ }^{1}$ NASA MUREP Scholar, NASA Langley Research Center, Hampton, VA 23681 \\ ${ }^{2}$ NASA Langley Research Center, Hampton, VA 23681 \\ ${ }^{3}$ Department of Mechanical and Aerospace Engineering, North Carolina State University, Raleigh, NC \\ 27695 \\ ${ }^{4}$ National Institute of Aerospace, Hampton, VA 23666 \\ ${ }^{5}$ NIFS Internship, NASA Langley Research Center, Hampton, VA 23681
}

\begin{abstract}
.
In this work, elastic microfences were generated for the purpose of measuring shear forces acting on a wind tunnel model. The microfences were fabricated in a two part process involving laser ablation patterning to generate a template in a polymer film followed by soft lithography with a two-part silicone. Incorporation of a fluorescent dye was demonstrated as a method to enhance contrast between the sensing elements and the substrate. Sensing elements consisted of multiple microfences prepared at different orientations to enable determination of both shear force and directionality. Microfence arrays were integrated into an optical microscope with sub-micrometer resolution. Initial experiments were conducted on a flat plate wind tunnel model. Both image stabilization algorithms and digital image correlation were utilized to determine the amount of fence deflection as a result of airflow. Initial free jet experiments indicated that the microfences could be readily displaced and this displacement was recorded through the microscope.
\end{abstract}

Introduction.

Understanding the behavior of turbulent airflow within the boundary layer is challenging due to the difficulty of making measurements without significantly disturbing the airflow. Shear stress measurements, in particular, are encumbered by dimensional constraints and sensor complexity. ${ }^{1}$ Although there are several traditional techniques that have a long history of use (hot wires, oil interferometry, calculation from pitot tube pressure measurements, floating elements balances etc.), these techniques may lack adequate precision and application flexibility which can encumber, if not compromise, their use for determining shear stress. ${ }^{1}$ Additionally, many of these techniques necessarily disrupt the airflow further compromising their results. Thus, there is a need to develop sensors and detection instrumentation that will be robust, accurate, easy-to-use, and reliable, while not disrupting the airflow.

Shear stress measurement techniques currently being developed consist of direct and indirect measurements. With direct measurements, the sensing element responds directly to shear forces. Typically, these approaches require precision fabricated micro electromechanical devices based on floating elements, interdigitated structures, or other complex geometries that take advantage of the sensitivity of the delicate mechanical structures to measure minute shear-induced loads, i.e., nanonewtons. ${ }^{2}$ Although these techniques have shown great promise, issues with sensor durability, high 
fabrication costs, and susceptibility to environmental conditions and contamination have hindered their development. Other direct measurement techniques have recently shown promise including whispering gallery mode sensors ${ }^{3,4}$ and fiber Bragg gratings. ${ }^{5}$ Several indirect techniques to measure shear stress have also been developed with two of the most promising being laser Doppler anemometry and small scale pressure drag forces. For small scale pressure drag forces, discrete elements affixed to a surface are displaced as a result of profile drag, which is subsequently related to shear forces acting on the surface. The size of the elements is indicative of the sensitivity of their displacement arising from forces acting near the surface, i.e., shear stress, with minimized contributions from airflow in the free stream. Brücker, Große, and Schröder have extensively investigated the application of micropost arrays for shear stress sensing in fluid flows, demonstrating the efficacy of this approach to conduct shear stress measurements. ${ }^{6-10}$ Similar designs have been utilized for various other applications. ${ }^{11-14}$

In this work, elastic surface topographies responsive to profile drag within the viscous sublayer (Figure 1) have been generated and implemented into a visual signal transduction device. The topographies consisted of "microfence" features with a height (z direction in Figure 1) that was approximately three times the thickness (flow direction in figure 1) and included stationary features referred to as fiducial marks, (long lines that are not displaced in the airflow) that enabled position referencing. The term "fence" is used here to differentiate the morphology of these structures from the cylindrical posts or pillars. A micropositioning device was designed to aid in alignment of the sensor surface to be flush with the airfoil surface and a versatile wind tunnel model is being fabricated for evaluation of this instrument prototype. Digital image stabilization and point tracking algorithms have been developed using data in preliminary wind tunnel experiments to improve the microfence displacement detection to sub-pixel resolution $(0.5 \mu \mathrm{m}$ per pixel).

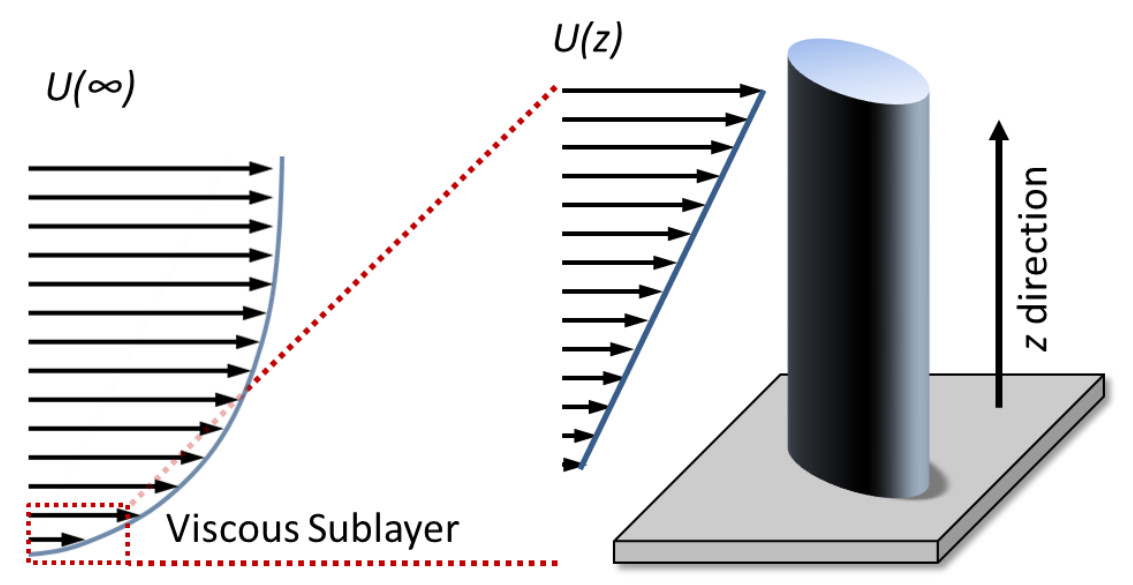

Figure 1. Microfence sensing elements were fabricated to remain within the viscous sublayer of subsonic turbulent airflow.

\section{Generation of Elastomeric Microfences.}

Sensing elements that protruded from a surface were chosen as the method to measure airflow interactions for a variety of reasons. First, as is described in detail below, the determination of shear forces from profile drag is well established. Next, the equations governing elastic bending of cantilevers 
are well known and can be adapted to the particular sensing element geometries fabricated here. The large market for commercial elastomers also provides a broad range of materials properties with elastic modulus values ranging from 0.1 to $3.0 \mathrm{MPa} .{ }^{15}$ Additionally the properties of a given 2-part silicone can be modified by changing the mixing ratio, adding diluent, or varying the cure conditions. ${ }^{16}$ Silicones are also commercially available, robust, chemically inert, and stable over a relatively broad thermal range, unlike materials used in other shear sensing approaches. Finally, other materials can be incorporated into the silicone seamlessly to extend sensing capabilities, such as fluorescent dyes used in this work, fiber optics, glass beads, ${ }^{6}$ etc.

Sensing Element Rationale and Limitations. Sensor resolution was defined by classic Euler-Bernoulli beam theory, which limited the maximum deflection of a sensing element to $10 \%$ of the height. Using this requirement, along with the intention to minimize disruption of the airflow by staying within the viscous sublayer, sensing element heights on the order of 50-100 $\mu \mathrm{m}$ were targeted. Therefore, deflections of no more than $10 \mu \mathrm{m}$ were preferred. In Euler-Bernoulli beam theory, deflection at the tip of a cantilevered cylindrical beam, $y$, can be described by:

$$
y=\frac{P L^{3}}{3 E I}
$$

where $L$ is the post length, $E$ is the Young's modulus, $P$ is the load, and $I$ is the area moment of inertia, which in this work was approximated as a rectangle with a fixed base of $b\left(I=1 / 3 b L^{3}\right)$. This expression assumes that the total bending load is at the fence tip. Other models, including distribution of the load along the fence length, did not significantly impact the calculated results. By describing the load as $P=D$, where $D$ is the profile drag, $D=1 / 2 \rho v^{2} S C_{D}$, the equation can be rewritten for the sensing elements described in this work by approximating the features as a triangular prism with the rectangular face as the frontal area, $S=L W$ :

$$
y=\frac{\rho v^{2} c_{D} L^{4} W}{6 E I}
$$

where $\rho$ and $v$ are the free stream density and velocity, respectively, $c_{D}$ is the drag coefficient and $W$ is the sensing element width. Considering a $100 \mu \mathrm{m}$ tall sensing element with an elastic modulus of $83 \mathrm{KPa}$ (this modulus corresponds to the modulus of the main silicone utilized in this work, Eccoflex ${ }^{\circledR} 0050$ supplied by Smooth-On Inc.), $1 \mu \mathrm{m}$ deflection would occur at a freestream velocity of $16.6 \mathrm{~m} / \mathrm{s}$. Viscous sublayer thickness and shear stress values were calculated for a variety of airflow velocities (Figure 2) anticipated to be utilized in this work using theoretical boundary solutions. ${ }^{17}$ These results guided the design for sensing element dimensions. 

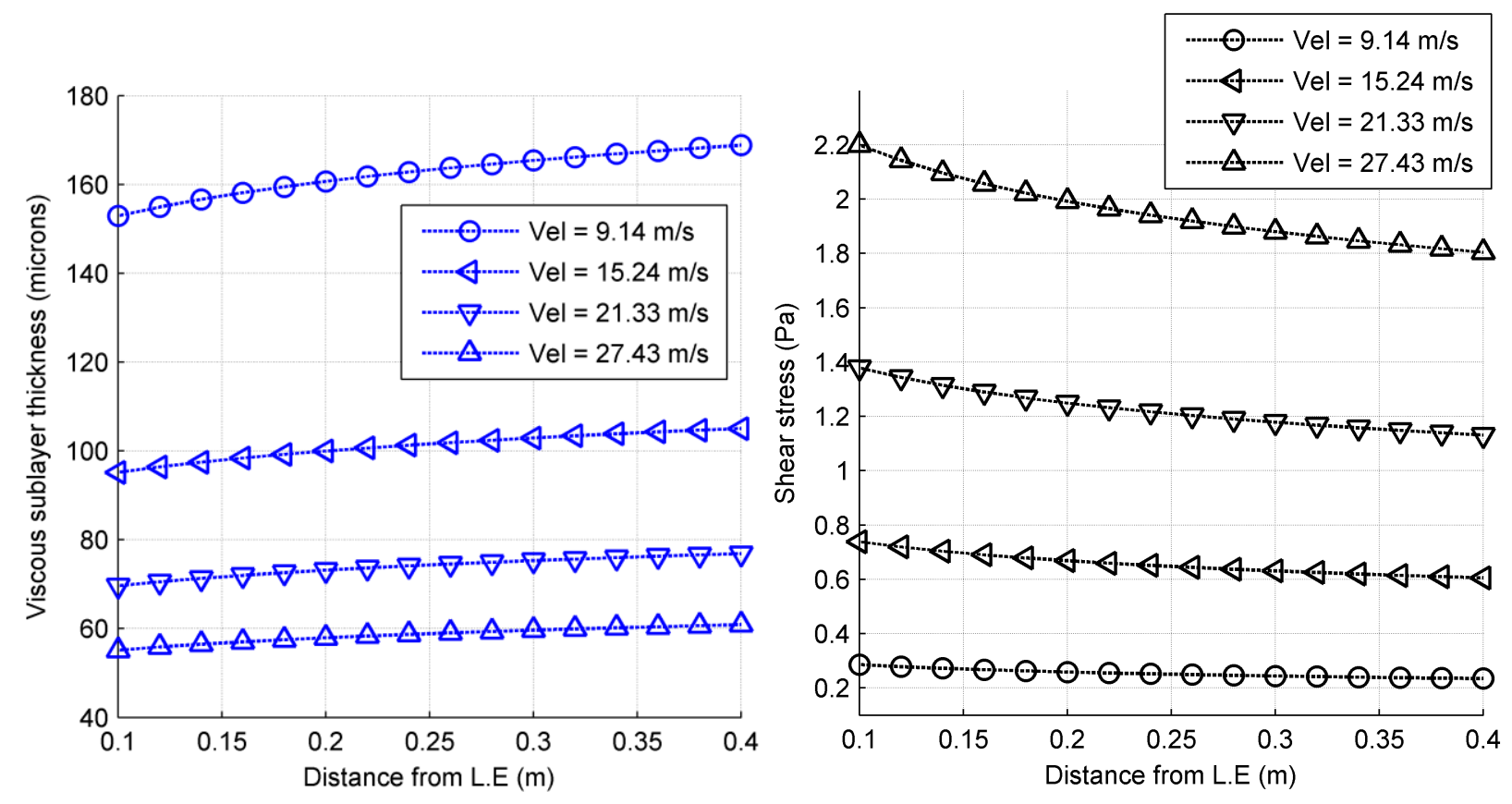

Figure 2. Calculated viscous sublayer thickness (left) and shear stress (right) for a flat plate model.

Fabrication of Microfence Arrays. In order to precisely measure sub-millimeter deflections, the maximum field-of-view of the microscope was relatively small $(0.64 \mathrm{~mm} \times 0.48 \mathrm{~mm})$ as described below. An array of sensing elements oriented at different angles, relative to the airflow, was designed to overcome this limitation (Figure 3A). The field-of-view only allowed for observation of a single position in the array (hereafter referred to as a die). In order to align the microscope with a single die, the instrument included a translation stage. Within a die, the spacing between microfences was maximized to prevent cross-talk due to disturbances in the airflow. Empty dies (no sensing elements) were included to further minimize interactions between adjacent dies in the array. A single sensor die consisted of two types of structures, sensing elements (short lines) and fiducial marks (long, crossed lines). The fiducial marks were included to enable accurate determination of sensing element deflection due to a difference in height. The fiducial marks had an aspect ratio (height/width) of much less than one which prevented their deflection in an airflow. 

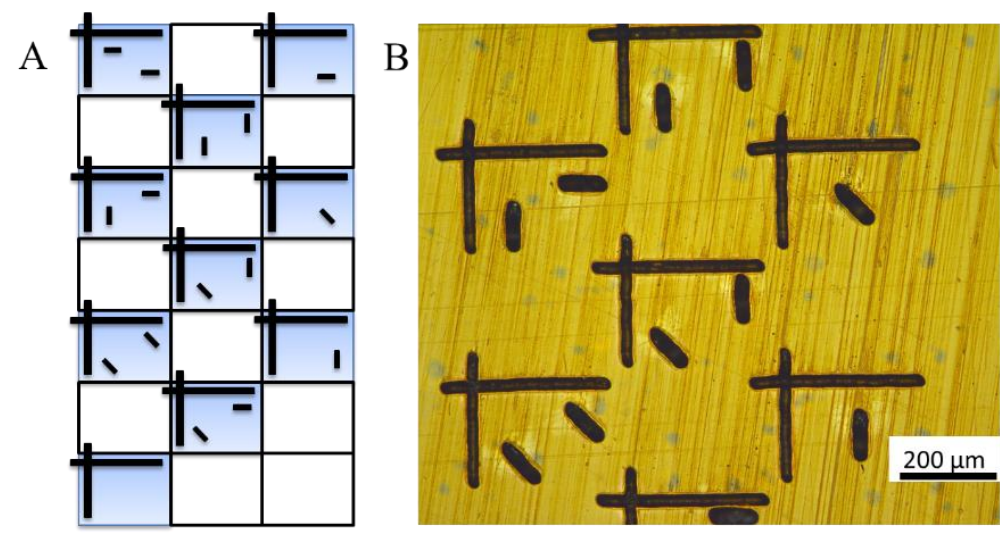

Figure 3. (A) Schematic of the $3 \times 6$ sensor array consisting of 10 sensor dies (blue) and 11 empty dies (white). (B) Optical micrograph of laser ablation patterned Kapton ${ }^{\circledR}$ HN. Fiducial marks are the long crossed lines and sensing elements are the smaller features.

A combination of direct-write and soft lithographic techniques were used to generate the microfence shear stress sensors. First, laser ablation using a frequency-tripled Nd:YAG laser (Coherent Avia, $\lambda=$ $355 \mathrm{~nm}$, average power $=2.5 \mathrm{~W}$, pulse width $=10 \mathrm{~ns}$ ) was utilized to direct-write templates. The laser machining system was provided by Photomachining Inc. with a galvanometer for focusing and steering the beam on the substrate. With this system, the marking speed and jump speed (moving between marked positions) are adjustable. Kapton ${ }^{\circledR} \mathrm{HN}$ was used as the ablation substrate because of its high absorption at $355 \mathrm{~nm}$ and its relatively low ablation threshold which is important for two reasons. First, it was important to ensure that photochemical and photophysical processes dominate relative to photothermal interactions. Second, as the power profile in each pulse follows roughly a Gaussian distribution, it was anticipated that being able to ablate a material at a relatively low power would enable smaller feature fabrication. Additionally, since this is a commercially available material, both precise control of substrate thickness and availability in a variety of thicknesses and grades improved process flexibility. A variety of laser ablation parameters were evaluated and several different approaches were identified as viable for making microfence sensing array templates. Ultimately, ablation of the fiducial marks at an average power of $40 \mathrm{~mW}$ with one pass and ablation of the microfence elements at an average power of $300 \mathrm{~mW}$ and 2 passes were used to generate several templates in both Kapton ${ }^{\circledR} \mathrm{HN}$ film and tape (Figure 3B). The following other parameters were kept constant: pulse frequency $=80 \mathrm{kHz}$, jump speed $=10.2 \mathrm{~cm} / \mathrm{s}(4 \mathrm{in} / \mathrm{s})$, mark speed $=2.5 \mathrm{~cm} / \mathrm{s}(1 \mathrm{in} / \mathrm{s})$, jog increment $=3 \mathrm{~mm}$.

Sensing elements were generated using soft lithography techniques. Silicone (EccoFlexx 00-50) was spincast (Headway Research Inc., PWM 32 Coater) on laser ablated Kapton ${ }^{\circledR}$ HN substrates that were affixed to $7.62 \mathrm{~cm}\left(3^{\prime \prime}\right)$ glass disks. Spin-casting was used to control the thickness and uniformity of the silicone layer being coated over the Kapton ${ }^{\circledR} \mathrm{HN}$ film. Six applications of the silicone on a single disk were needed to prepare films 0.5 to $1 \mathrm{~mm}$ in thickness which provided the necessary mechanical properties for demolding and handling. The uncured silicone was heated for two minutes between each application to partially cure the film. Alternatively, curing the silicone at room temperature for at least 15 minutes resulted in facile removal from the template, relative to the elevated cure temperature. The resultant silicone surfaces were characterized using scanning electron mciroscopy (SEM) using a JEOL 5600 (Figure 4). The surfaces were sputter-coated with 3-6 nm of Pd:Au to increase conductivity. The reusability of 
the laser ablation patterned Kapton ${ }^{\circledR} \mathrm{HN}$ template was evaluated with 3 iterations of patterned silicone surfaces being generated with nominal changes in sensor shape and morphology as determined by SEM.
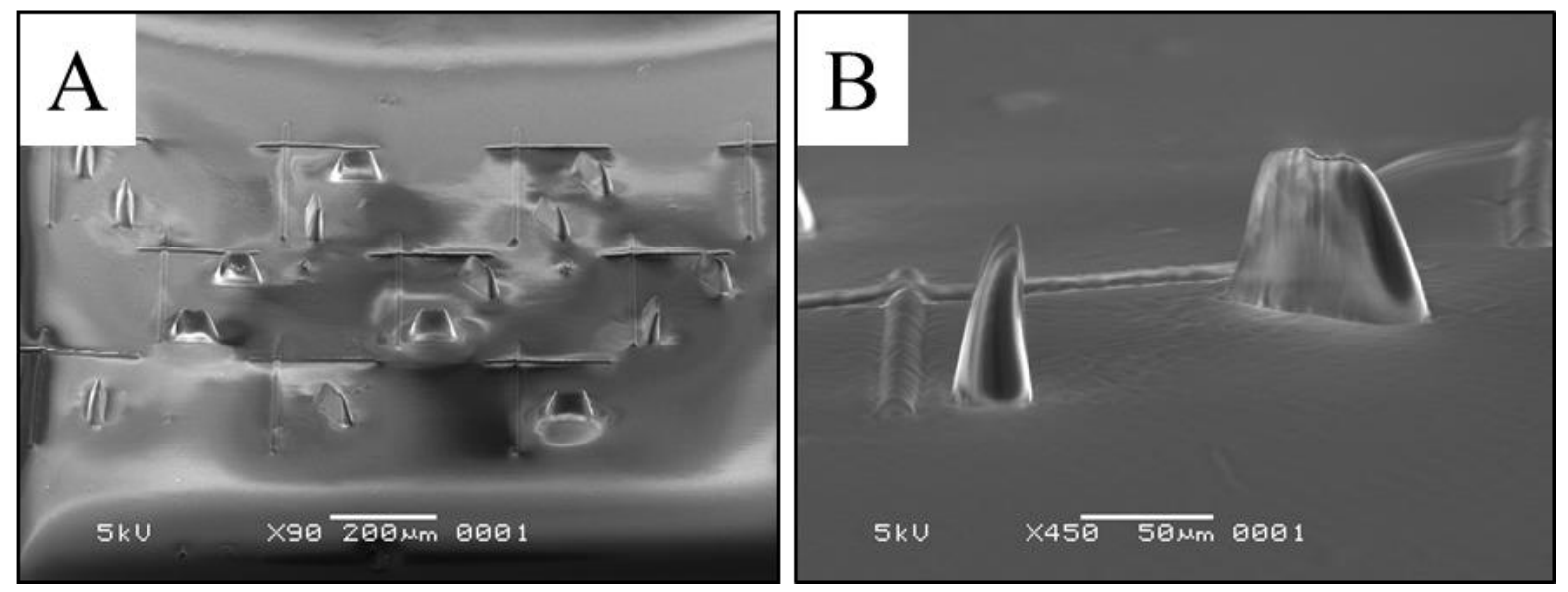

Figure 4. The wide view showing multiple dies (A) and a tight view showing a single die (B) both have a tilt-view angle of 45 degrees. Laser paramters were $300 \mathrm{~mW}$ and 2 passes to prepare the fences. Surface color variation is due to charging effects.

Contrast Enhancement using Rhodamine B. With the use of optical detection, differentiation of the sensing elements and fiducial marks from the substrate would result in a significantly greater signal-tonoise ratio. To that end, several methods were evaluated to "stain" the tips of the microfences and the fiducial marks. Two main approaches were evaluated: staining the microfences by "through-ablation" of the template, and deposition of dye on the template prior to silicone casting. The most straightforward approach was initially to ablate through the Kapton ${ }^{\circledR} \mathrm{HN}$ using $75 \mu \mathrm{m}$ (3 mil) thick film. The template was removed from the glass substrate after casting and curing the silicone. A methanol solution of rhodamine $B$ was deposited on the underside of the template with the silicone tips of the microfences also exposed resulting in adsorption of dye by the exposed silicone features. Although this approach was promising, there was no way to stain the fiducial marks that were not ablated through the film. An alternative approach was to deposit a rhodamine B solution on the template surface prior to silicone molding followed by removal of excess solution using a squeegee device. Although initial experiments using the relatively volatile ethanol/water solution were not successful, changing to a toluene solution with a few drops of methanol to solubilize the dye was successful. Using an inverted fluorescence microscopy (Olympus, IX71), the staining of the microfence tips was verified (Figure 5). The fiducial marks do not appear to be stained, which could arise from the relatively shallow nature of these features compared to the microfences. Future work will involve modification of the fiducial mark heights to enable staining. 

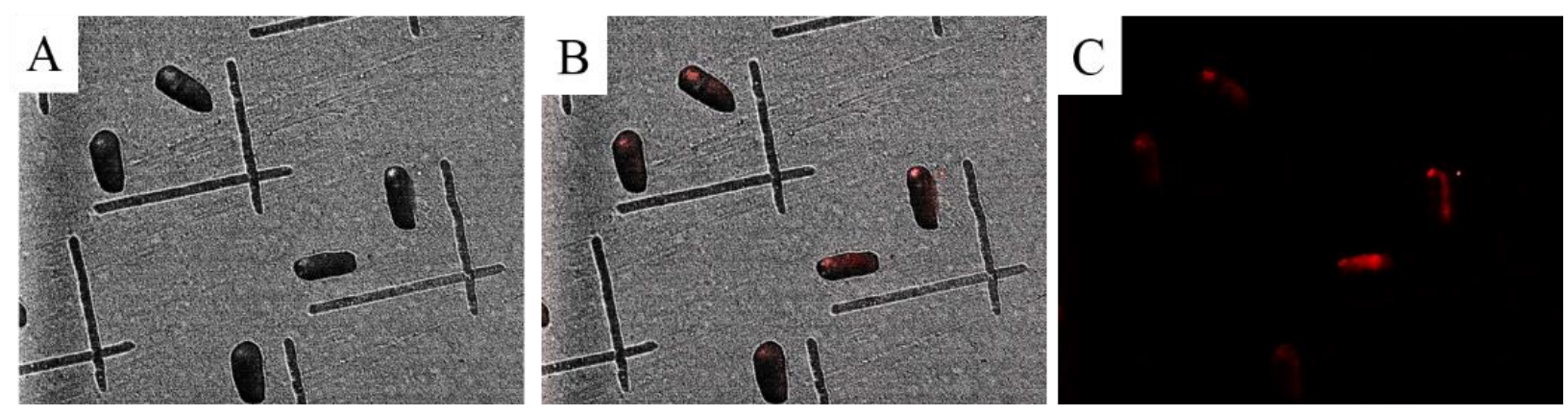

Figure 5. (A) Absorption and (C) fluorescent emission micrographs of rhodamine $B$ stained silicone microfence arrays. The image in (B) is a composite of the images in (A) and (C).

\section{Wind Tunnel Testing}

Instrument Components. Optical visualization was determined to be the most promising means of measuring deflection in the microfence elements. Therefore, an instrument was designed utilizing optical microscope hardware to provide lighting and resolution necessary to detect deflection of sensing elements. Integration into a wind tunnel model also guided prototype design. By using a single axis instrument with in-line lighting elements, the footprint of the instrument and dimension of any extremities were minimized (Figure 6A). The instrument consisted of a microscope objective connected to an in-line light source that illuminated the sensing element positioned above the objective. A chargecoupled device (CCD) camera mounted to focusing optics was connected to the lighting element. (Either a white light or UV-light can be used as the illumination source, which enables specialized contrast enhancement techniques, as described above.) A picture of the instrument prototype with a white light source incorporated is shown in Figure 6B. The instrument is inverted relative to the intended orientation in the wind tunnel. A $10 \times$ microscope objective with a long working distance was utilized to provide the necessary resolution and configuration flexibility for this instrument.
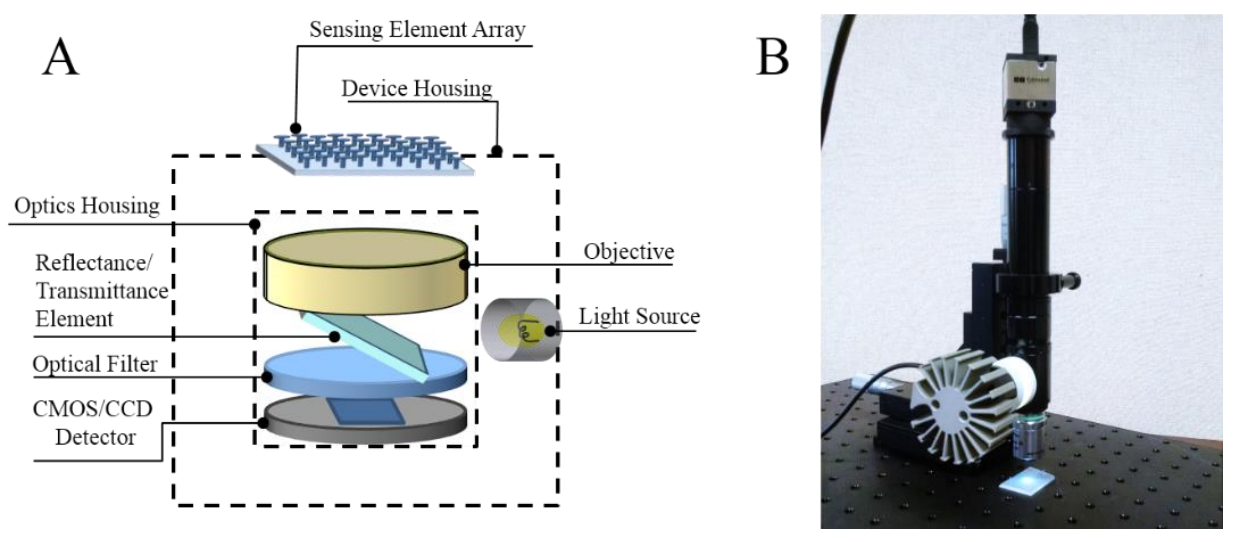

Figure 6. (A) Schematic and (B) photograph of the shear stress sensor prototype instrument. Note, the instrument in $(B)$ is inverted relative to the installation in the wind tunnel model at NCSU. 
Wind tunnel model. Characterization of the microfence shear stress sensors under real wind flow conditions required experimental testing in a wind tunnel. The North Carolina State University (NCSU) subsonic wind tunnel offered the capability to evaluate the response of the microfence sensors by comparing the microfence deflections with aerodynamic measurements from several traditional approaches. A flat-plate model with a rounded leading edge, resembling a thin airfoil, was proposed for the integration and testing of the microfence array. The sensors were installed on a quartz disk (approximately $3.2 \mathrm{~cm}\left(1.25^{\prime \prime}\right)$ in diameter) that was flush mounted on the surface of the flat-plate airfoil model. The microscope (Figure 6 B) was housed in a faired support structure. A first-generation flat-plate model was designed as a first iteration to conduct preliminary tests in the wind tunnel with the microfence samples. The objective of this first-generation model was to gain more information regarding the integration of the microfence sensor setup in the wind tunnel and gather more insight towards designing an optimized final model capable of testing the microfence arrays under different flow scenarios.

First Generation Model. The first test apparatus was a flat plate fabricated from medium density fiberboard (MDF). The flat plate was $40.6 \mathrm{~cm}\left(16^{\prime \prime}\right)$ in chord, $81.3 \mathrm{~cm}\left(32^{\prime \prime}\right)$ in span and $1.9 \mathrm{~cm}\left(3 / 4^{\prime \prime}\right)$ thick. It was mounted horizontally to span from wall to wall, so as to achieve nearly two-dimensional flow on the plate. A semi-circular leading edge was attached to the front edge of the flat plate and the microfence array housing was positioned at a streamwise station of $22.9 \mathrm{~cm}\left(9^{\prime \prime}\right)$ from the leading-edge of the flat plate. This flat-plate model was supported by a cylindrical PVC support structure with inner diameter of $30.5 \mathrm{~cm}\left(12^{\prime \prime}\right)$, which provided sufficient space to house the camera assembly and related equipment necessary for the measurement of microfence deflection. Additionally, the flat-plate model had an extended trailing-edge flap attachment. This movable flap provided the capability to adjust the overall pressure distribution over the flat plate ensuring that the stagnation point was positioned on the upper surface of the flat plate. The different parts of the crude model are shown together unassembled in Figures 7 and 8 .
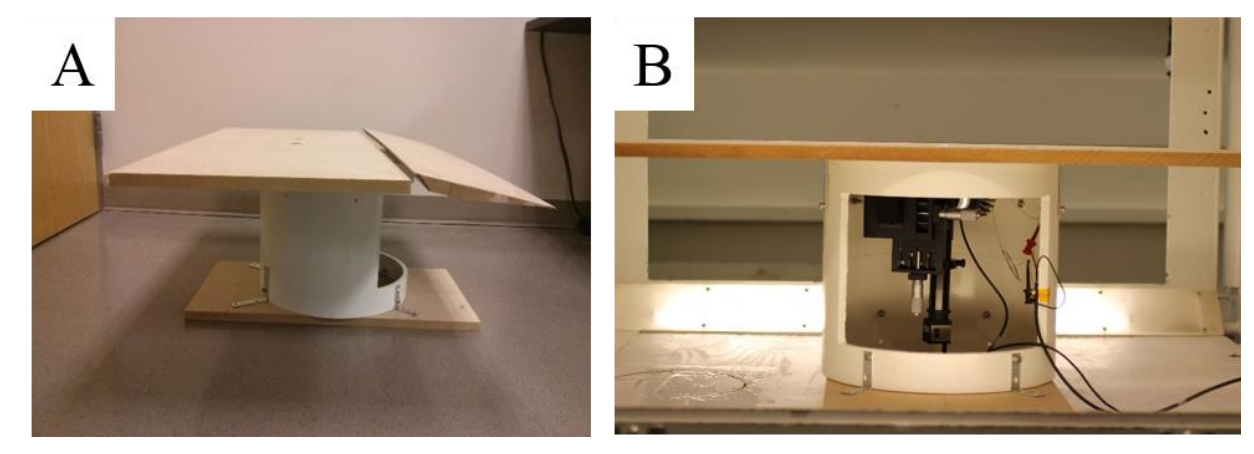

Figure 7. (A) Side view of the first-generation flat-plate model and the trailing edge flap unattached. (B) The first-generation model assembled inside the wind tunnel test section along with the camera setup.

Refined model. The final refined model for the test setup will be the next step towards experimental testing. The flat plate in this setup is proposed to be a custom designed thin airfoil with flap at the trailing edge (Figure 8). The complete model would be fabricated using stereo-lithography. A faired support structure, designed to support the flat-plate model, will have internal access for installing the camera assembly to monitor the microfence sensors from below during the experiments. This model will have a 
trailing-edge flap that can be adjusted to control the stagnation point at the leading-edge of the model. This control will ensure that there are no suction peaks at the leading edge. Various adjustable airfoil/cylinder models will be installed above the flat-plate model with the capability to adjust the vertical height and the angle of attack. The model will be rapid-prototyped with integral pressure taps and tubing. The information from these pressure measurements can provide a clear understanding of the flow and they can also be effectively used with standard boundary layer calculations to estimate the state of the boundary layer at the sensor location. Direct measurement of shear stress can be determined from a conventional shear stress sensor with wired signal transduction. Additionally, hot-wire measurements of the boundary-layer flow in the vicinity of the microfence sensors will be conducted by adapting an existing $x-y$ traverse system for positioning. These hot wire measurements provide another approach to deduce the wall shear stress. Such an extensive setup enables testing the effectiveness of the microfence sensors under a variety of flow conditions: laminar and turbulent boundary layer under favorable and adverse pressure gradient; separated boundary layers; and separation and reattachment points.
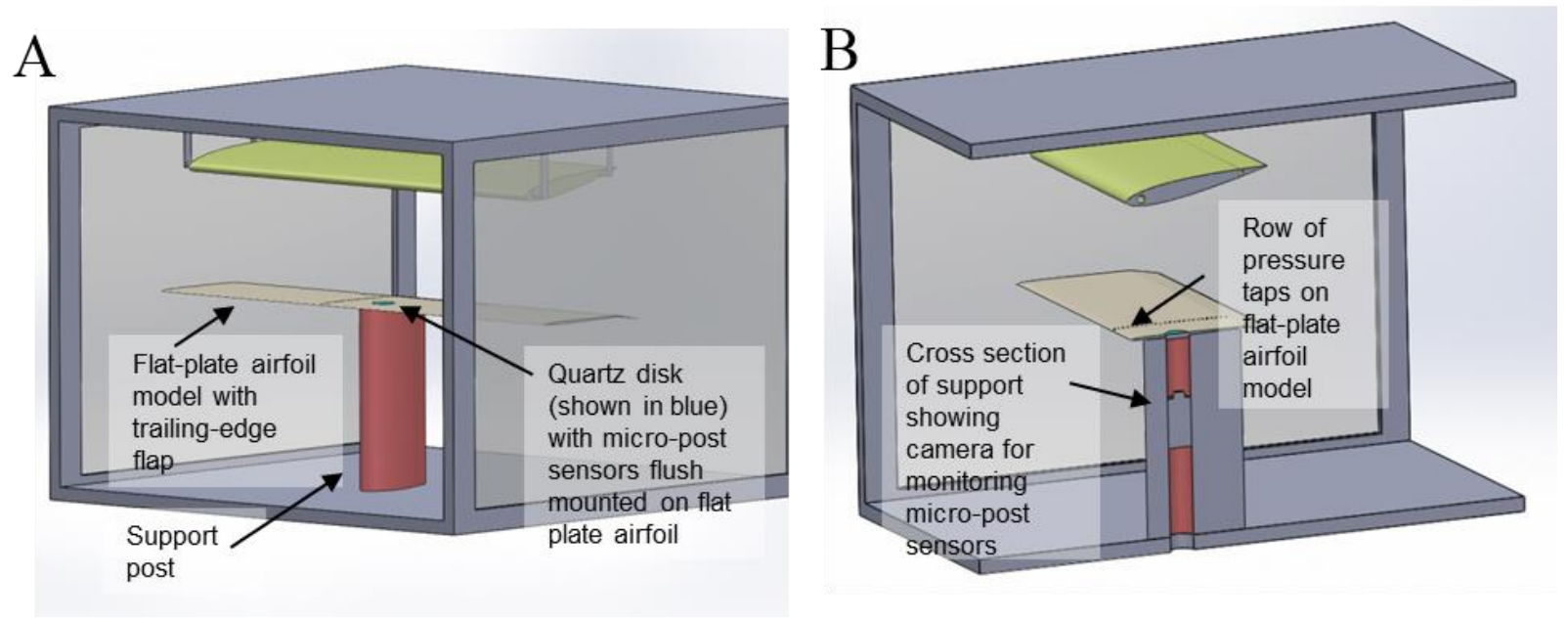

Figure 8. Experimental layout for making shear stress measurements in the wind tunnel at NCSU showing a complete view (A) and cross-section view (B). The airfoil mounted at the top of the wind tunnel can be translated vertically to change the separation distance

from the flat plate and the sensor position can be changed stream-wise to make measurements at various locations along the flat plate.

Design of elevator mechanism. To accurately measure shear stress acting on the wind tunnel model, it was critical that the surface of the sensor disk be aligned with the top surface of the flat plate. This prevented disturbance of the flow and protrusion of the sensors above the viscous sublayer. An elevator mechanism was designed for precision alignment and adjustment of the sensor alignment with the flat plate. The mechanism enabled alignment adjustments to be made quickly without removal of the flat plate from the fairing. The mechanism was designed using Autodesk Inventor Professional 2015 (Autodesk, Inc., San Rafael, CA). Adjustments were made by applying torque to a shaft located arbitrarily away from the flat plate (Figure 9).

An input shaft, located outside of the fairing, turned a worm drive, which rotated a gear and transfered this rotational motion into translational motion of the sensors. The worm/gear housing utilized a key 
which prevented rotation of the sensor while allowing translation. The assembly was located inside the flat plate model and the microscope's objective was located inside the hollow, translation shaft.

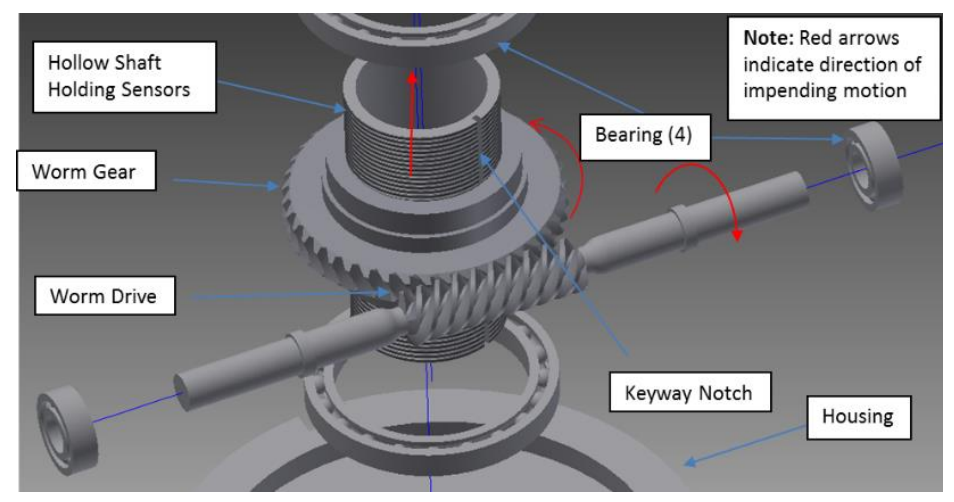

Figure 9. Exploded view of the elevator mechanism, designed to integrate into the refined model, enabling refined vertical positioning of the sensor elements to prevent airflow disruption.

\section{Fence Deflection Capture, Processing, and Transduction.}

Fence deflection and inspection experiments were initially tested outside of the wind tunnel using a free jet of compressed gas to create flow around the sensors. Video of fence deflection were collected using the microscope shown in Figure $6 \mathrm{~B}$. Figure 10 shows three frames collected from a video where the jet was off, on at a moderate flow rate, and on at the full flow rate. Deflection of the fence is clearly observed by eye and the distance between the tip and the lower fiducial can be measured in the captured images as indicated with the orange lines. The distance between the fiducial mark and the fence tip decreased by about $14 \%$ with the moderate jet and $19 \%$ with the full jet.

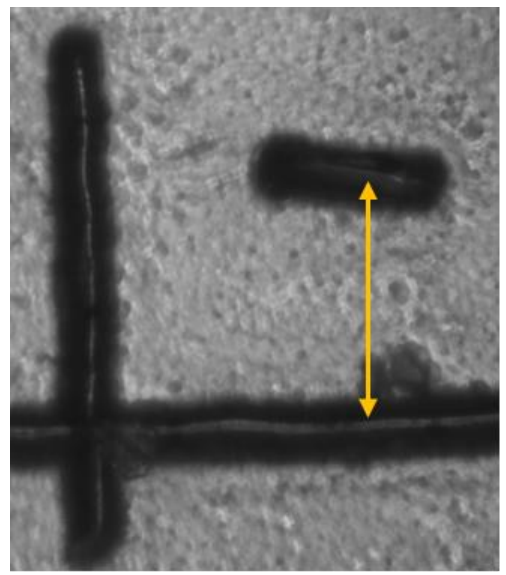

No jet

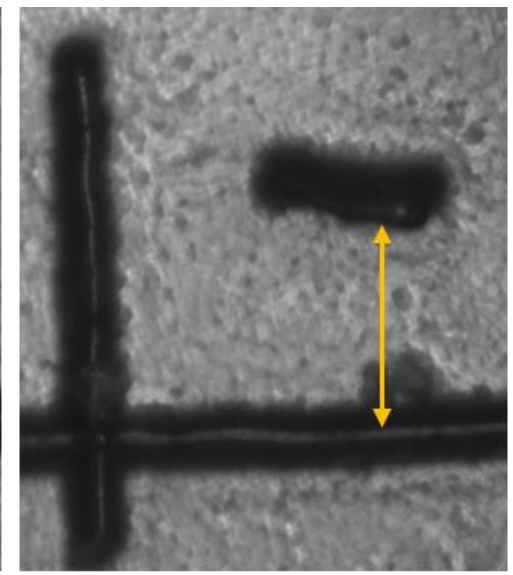

Moderate jet

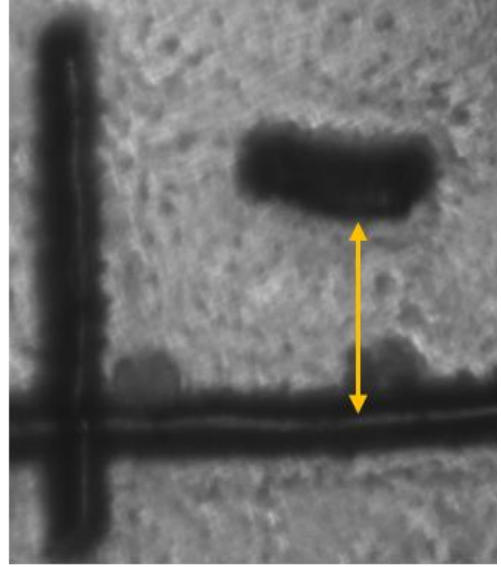

Full jet

Figure 10. Sensor elements deflecting due to impingement with flow from a free jet.

Preliminary wind tunnel tests were conducted by mounting the microfence arrays flush on the surface of the first-generation test model. Figure 11 shows the glass disk, with the sensor elements, mounted in the 
flat plate and also shows the image of the microfence structure under investigation from one of the test cases. Tests were done for different freestream velocities to identify the microfence deflection under a range of shear stress cases. A qualitative estimate of the expected shear stress and skin friction coefficient was made using the theoretical boundary flow solutions for laminar and turbulent flow regimes. Laminar flow was achieved at the sensor location for a Reynolds number less than 500,000, while measures were taken to attain turbulent flow boundary layer at lower velocities by tripping the flow.

Data for these experiments was recorded in the form of videos captured by the CCD camera. The camera was able to identify the microfence tip with the aid of conventional microscopy technology, attached inline and focused on the microfences. The camera setup was installed on the lower surface of the flat plate and was housed in the supporting structure to protect it from the incoming flow inside the wind tunnel. Care was taken to ensure that the entire experimental setup including the flat plate was not physically in contact with the wind tunnel test section. This was an essential step taken to eliminate the effects of any mechanical vibration present in the wind tunnel walls.
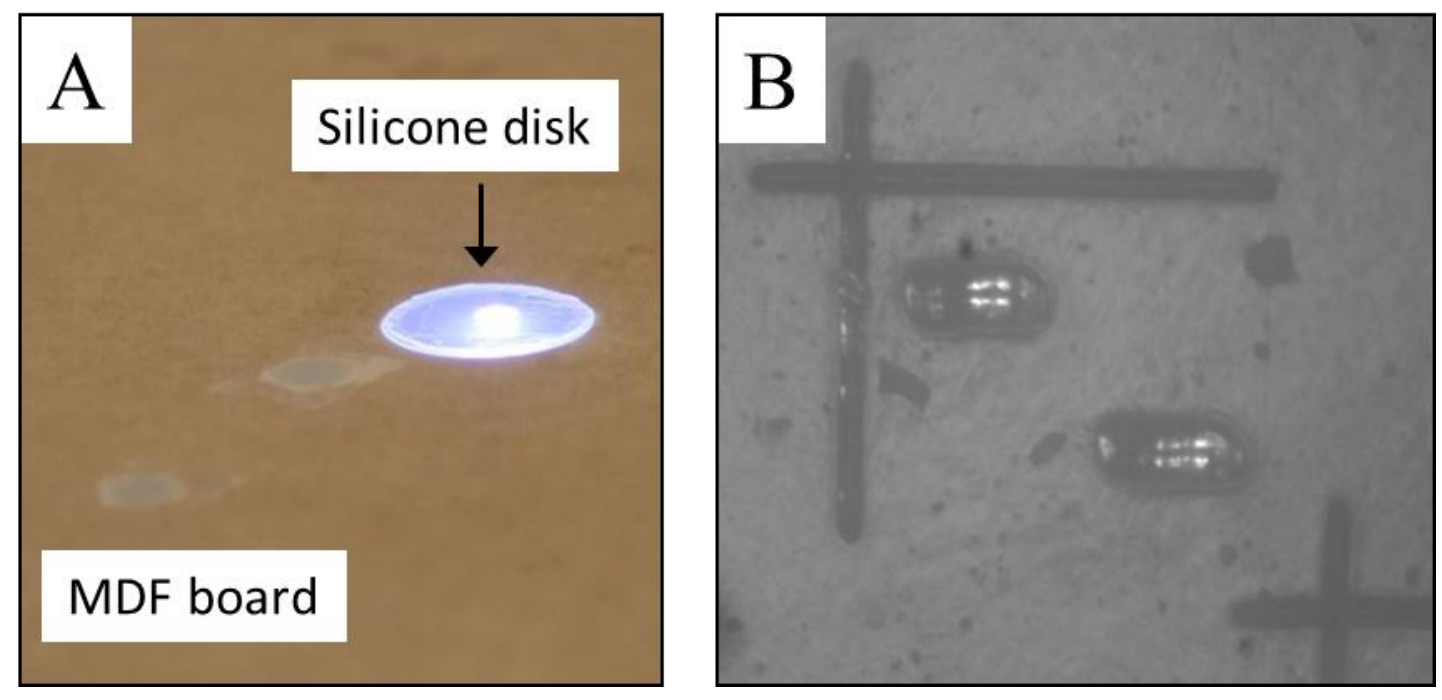

Figure 11. (A) Silicone sensors on glass disk mounted in the MDF, flat-plate model and (B) a topdown image of and senor die collected by the microscope installed in the fairing.

Although mechanical vibrations were prevented, flow induced vibrations of the flat-plate model were still observed during the experimentation. As a result, the vibration effect interfered with the videos recorded by the camera. Determination of microfence deflection was done by employing a digital image correlation (DIC) algorithm, which extracts and compares individual images from a video sequence. Therefore, image pre-processing was required to mitigate the effect of flow-induced vibration during the correlation procedure.

Image Stabilization by Feature Tracking. The image stabilization method employed here relied on tracking the fiducial marks as fixed features in all the frames of the recorded video and then realigning and resizing the images based on the locations of these fiducials. Images extracted from the entire video sequence were first enhanced to achieve greater contrast using the image processing toolbox in MATLAB (Figure 12). The intensity values of the original grayscale images were mapped to new values such that $1 \%$ of data is saturated at low and high intensities of the original grayscale image (Figure 12B). This step increased 
the contrast of the images. The images were still in grayscale format, i.e. every pixel location had an associated intensity value. Next, the images were converted to binary form, such that the pixels had a value of either 1 (white) or 0 (black). This step enables the identification of different sized regions from the image data. The feature tracking and realignment process was conducted as a batch process and all the frames of the recorded video were assessed.

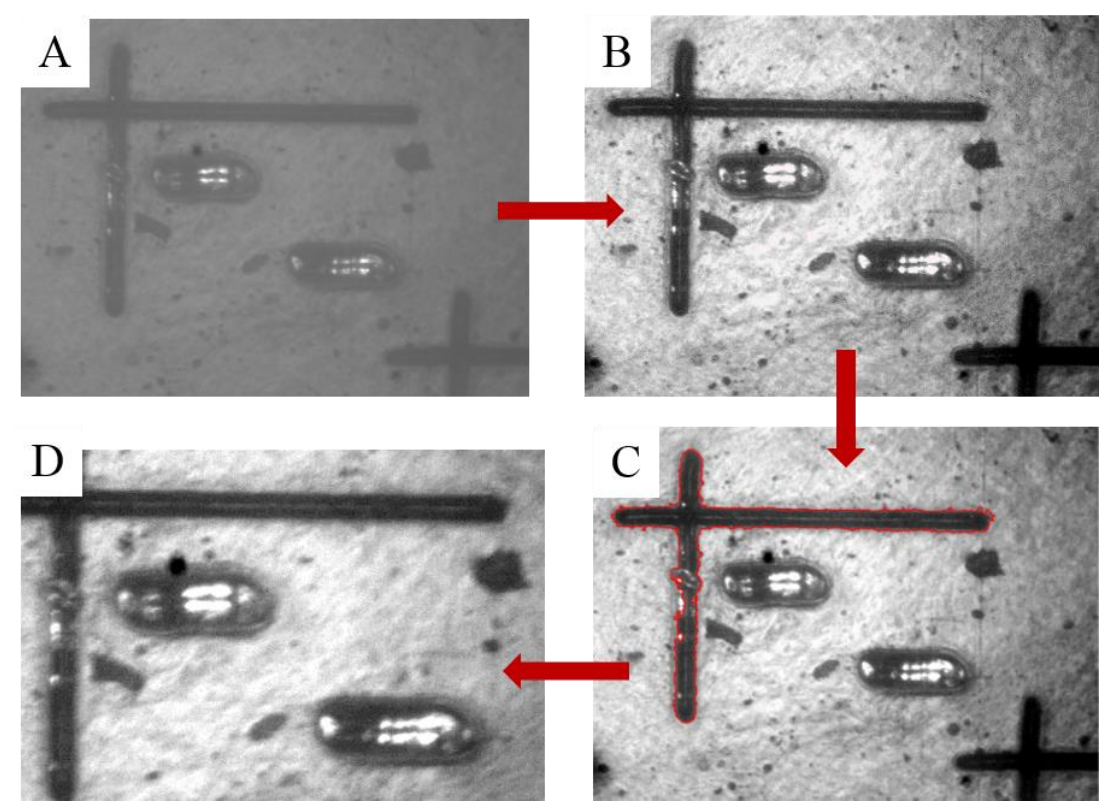

Figure 12. (A) A raw image captured by the camera. (B) An enhanced version of the image in (A) with greater contrast. (C) Reference feature, in red, were tracked by the algorithm. (D)The final re-sized and re-aligned image based on the tracked reference.

Image Tracking Algorithm Based on Digital Image Correlation (DIC). DIC is an optical method that employs correlations to measure shifts in datasets and this technique was applied to accurately measure 2D and 3D changes in images. ${ }^{18,19}$ This method finds potential application in a wide range of areas that involve the measurement of deformation, displacement, strain and optical flow. DIC techniques have shown greater benefits compared to manual measurement methods in micro- and nano-scale mechanical testing. The fundamental principle behind this method is to discretize a reference image into blocks or subsets of pixels and then compare these pixel blocks with a subsequent image from the sequence, recorded at different instances of time, using correlation functions. This correlation coefficient can be determined by examining the pixel intensity between the corresponding pixel blocks and extracting a displacement mapping function to relate the images. The nonlinear optimization methods intrinsic to DIC provide the ability to attain sub-pixel resolutions in the analysis of images obtained using conventional photography/microscopy technologies.

A similar algorithm was employed in this research to track the deflection of the microfence tip under the influence of shear stress. The DIC and tracking code utilized in this research was developed by Dr. Chris Eberl at Johns Hopkins University and is available as an open access tool for the research community. ${ }^{20}$ The code was programmed in MATLAB and uses the image processing and optimization toolboxes. Figure 
$13 \mathrm{~A}$ shows a grid of the pixels covering one microfence and Figure $13 \mathrm{~B}$ presents a plot of the corresponding displacement of these pixel over the entire video sequence.
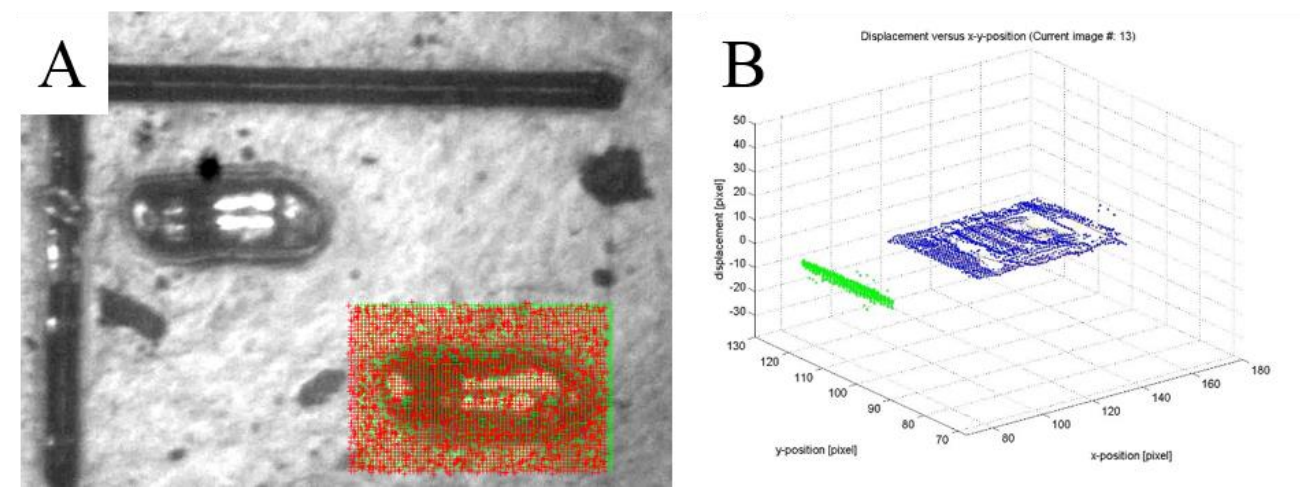

Figure 13. (A) The grid used for analyzing the microfence deflection and (B) the plot of the corresponding pixel displacements. The green is the projection of all the blue points onto a single line.

\section{Conclusions.}

The use of microfence sensing elements for determination of shear stress through relation to profile drag requires both precise control of microfence geometry and precise determination of microfence deflection. Although more accurate methods, such as photolithography, would provide greater control of the microfence shape, laser ablation patterning proved invaluable with regards to the flexibility to change the sensing element dimensions, array die lay-out, and the proximity of one array to the next. With relatively straightforward algorithms and accepted practices used regularly in digital image correlation, the ability to track a large number of pixels relative to either stationary objects within the field of view or the original pixel location has enabled precise measurements of fence deflection. Flow separation and other unforeseen challenges have prevented observation of fence deflection in the wind tunnel environment. However, exposure of the microfences to a jet did result in deflection that validated the functionality of the image tracking algorithm. Likewise, the use of a two-part silicone enabled introduction of fluorescent dye that should further improve differentiation of the microfence tips from the substrate. Ultimately, the use of these robust shear stress sensors may enable measurement of shear forces under conditions that would otherwise be impossible to ascertain this data in.

\section{References.}

(1) Naughton, J. W. and Sheplak, M., "Modern Developments in Shear-Stress Measurement," Prog. Aerospace Sci., 38, 2002, pp. 515-570.

(2) Meloy, J., Griffin, J., Sells, J., Chandrasekaran, V., Cattafesta, L. and Sheplak, M., "Experimental Verification of a Mems Based Skin Friction Sensor for Quantitative Wall Shear Stress Measurement," 41st AIAA Fluid Dynamics Conference and Exhibit, Honolulu, Hawaii, 2011.

(3) Ioppolo, T., Ayaz, U. K. and Otugen, M. V., "Performance of a Micro-Optical Wall Shear Stress Sensor Based on Whispering Gallery Mode Resonators," 47th AIAA Aerospace Sciences Meeting, Orlando, FL, 2009.

(4) Zamanian, A. H. and loppolo, T., "Investigation of a Photonic Wall Pressure and Shear Stress Sensor," 53rd AIAA Aerospace Science, Kissimmee, Florida, 2015. 
(5) Soh, C.-K., Yang, Y. and Bhalla, S. Smart Materials in Structural Health Monitoring, Control and Biomechanics; Zhejiang University Press: Hangzhou, China, 2012.

(6) Grobe, S., Schroder, W. and Brucker, C., "Nano-Newton Drag Sensor Based on Flexible MicroPillars," Meas. Sci. Technol., 17, 2006, pp. 2689-2697.

(7) Schmitz, G. J., Brucker, C. and Jacobs, P., "Manufacture of High-Aspect-Ratio Micro-Hair Sensor Arrays," J. Micromech. Microeng., 15, 2005, pp. 1904-1910.

(8) Grobe, S. and Schroder, W., "Mean Wall-Shear Stress Measurements Using the Micropillar ShearStress Sensor Mps3," Meas. Sci. Technol., 19, 2008, pp. 015403.

(9) Grobe, S. and Schroder, W., "Dynamic Wall-Shear Stress Measurements in Turbulent Pipe Flow Using Micro-Pillar Sensors Mps3," Int. J. Heat Fluid Flow, 29, 2008, pp. 830-840.

(10) Notterbrock, B., Klaas, M. and Schroder, W., "Improvement of the Measurement Range of the Micro-Pillar Shear-Stress Sensor Mps3," 28th AIAA Aerodynamic Measurement Technology, Ground Testing, and Flight Testing Conference, New Orleans, Louisiana, 2012.

(11) Li, B., Xie, L., Starr, Z. C., Yang, Z., Lin, J.-S. and Wang, J. H.-C., "Development of Micropost Force Sensor Arrays with Culture Experiments for Determination of Cell Traction Forces," Cell Motil. Cytoskeleton, 64, 2007, pp. 509-5018.

(12) Xu, T., Zhu, N., Xu, M. Y.-C., Wosinksi, L., Aitchison, J. S. and Ruda, H. E., "Pill-Array Based Optical Sensor," Optics Express, 16, 2010, pp. 5420-5425.

(13) del Campo, A., Greiner, C. and Arzt, E., "Contact Shape Controls Adhesion of Bioinspired Fibrillar Surfaces," Langmuir, 23, 2007, pp. 10235 - 10243.

(14) Geim, A. K., Dubonos, S. V., Grigorieva, I. V., Novoselov, K. S., Zhukov, A. A. and Shapoval, S. Y., "Microfabricated Adhesive Mimicking Gecko Foot-Hair," Nat. Mat., 2, 2003, pp. 461-463.

(15) Lotters, J. C., Olthuis, W., Veltink, P. H. and Bergveld, P., "The Mechanical Properties of the Rubber Elastic Polymer Polydimethylsiloxane for Sensor Applications," J. Micromech. Microeng., 7, 1997, pp. 145147.

(16) Schneider, F., Fellner, T., Wilde, J. and Wallrabe, U., "Mechanical Properties of Silicones for Mems," J. Micromech. Microeng., 18, 2008, pp. 065008.

(17) Schlichting, H. Boundary-Layer Theory; McGraw Hill: New York, 1979.

(18) Keating, T. J., Wolf, P. R. and Scarpace, F. L., "An Improved Method of Digital Image Correlation," Photogrammetric Eng. Remote Sens., 41, 1975, pp. 993-1002.

(19) Sutton, M. A., Orteu, J. J. and Schreier, H. W. Image Correlation for Shape, Motion and Deformation Measurements; Springer: New Yotk, 2009.

(20) Eberl, C., Digital Image Correlation and Tracking. http://www.mathworks.com/matlabcentral/fileexchange/12413-digital-image-correlation-and-tracking (March 16, 2015). 